\title{
EL LIDERAZGO EN LA GESTIÓN EDUCATIVA, UNA PROEZA DEL DIRECTOR EDUCATIVO DESDE LA PERSPECTIVA LATINA
}

Para citar este artículo puede utilizar el siguiente formato:

Iván Leonardo Medina Alvarado (2020): "El liderazgo en la gestión educativa, una proeza del director educativo desde la perspectiva latina", Revista Caribeña de Ciencias Sociales (julio 2020). En línea:

https://www.eumed.net/rev/caribe/2020/07/gestion-educativa.html

\section{Resumen}

Este escrito es generado a partir de una revisión bibliográfica, con la cual se confecciona un documento que comprende cómo el contexto latinoamericano denota ciertas particularidades en el manejo de un centro educativo; dando a conocer cuatro pilares fundamentales en los que el liderazgo se constituye como un elemento de vital importancia en la gestión educativa, siendo estos pilares: a) La gestión del cambio en la organización educativa, siendo una paradoja con la cual el director de un centro educativo debe hacer frente día a día desde su labor, b) Incidencia de la comunicación en el liderazgo educativo, siendo representativo el impacto que tiene la comunicación para con la gestión de las relaciones humanas, c) El manejo de conflictos en el entorno académico, como elemento diferenciador de la gestión educativa y de la sobrevivencia de una institución de educación, y d) La cultura organizacional en el centro educativo, este último pilar presenta un análisis sobre los aspectos a considerar en el fortalecimiento de la cultura e identidad de la organización educativa. Por último, se presentan las conclusiones a las que se llega posterior a este análisis y los referentes bibliográficos.

Palabras clave: Liderazgo docente, gestión educativa, organización educativa, dirección educativa, administración educativa, gestión escolar.

\author{
Abstract \\ ${ }^{1}$ Doctorando PhD en Educación (UBC - México) \\ Magister en Dirección y Administración de Empresas MBA (UNIR - España) \\ Especialista en Gerencia del Talento Humano y Riesgos Ocupacionales (UNITEC - Colombia) \\ Administrador de Empresas (CUN - Colombia) \\ Docente de pregrado Universidad Santo Tomás (USTA - Colombia) \\ Docente de pregrado programa Administración de Empresas Fundación Universitaria del Área Andina (FUAA - Colombia) \\ Docente de posgrado MBA - Middlesex University London / KMU Education Business School (Curacao) \\ ivanmedina@ustadistancia.edu.co, imedina6@areandina.edu.co, ivan.medina.alvarado@gmail.com.
}


This document is generated from a bibliographic review, with which a document is prepared that understands how the Latin American context denotes certain peculiarities in the management of an educational center; making known four fundamental pillars in which leadership is constituted as an element of vital importance in educational management, these pillars being: a) The management of change in the educational organization, being a paradox with the quality the director of a center $b$ ) Incidence of communication in educational leadership, being representative the impact that communication has for the management of human relations, c) The management of conflicts in the academic environment, as a differentiating element of educational management and survival of an educational institution, and d) The organizational culture in the educational center, this last pillar presents an analysis of the aspects to be considered in strengthening the culture and identity of the educational organization. Finally, the conclusions reached after this analysis and the bibliographic references are presented.

Keywords: Teacher leadership, educational management, educational organization, educational direction, educational administration, school management.

\section{EL DIRECTOR EDUCATIVO COMO GESTOR DEL CAMBIO Y LA TRANSFORMACIÓN}

Para comprender la labor del directivo en el sector educativo, resulta más que necesario identificar alternativas para conocer en detalle los aspectos que determinan esta labor, es así como este documento es generado con la intención de encontrar los elementos fundamentales a considerar en este ejercicio, desde la interacción que este sujeto tiene para con toda la comunidad educativa y los diferentes grupos de interés que se traducen en la sociedad en general.

Cabe mencionar, que este análisis es generado a partir de la información recolectada entorno al escenario latinoamericano, toda vez, que es claro el identificar que Latinoamérica cuenta con patrones y elementos únicos que no pueden ser abordados desde la misma mirada de resto del mundo, razón por la cual se abordan algunas situaciones que se viven en los diferentes centros de educación de países como Colombia, México, Argentina, Chile, Costa Rica, Brasil entre otros.

La importancia de este documento, es evidenciable cuando el escenario latinoamericano en pleno siglo XXI está experimentando múltiples cambios de todo tipo, pasando por el escenario social, económico, político y ambiental, para lo cual, la información recolectada es compilada desde una revisión bibliográfica, que da cuenta de los movimientos sociales y la incidencia de estos en los procesos educativos de la dirección educativa desde todo nivel, demostrando de este modo, la afectación que tienen estos elementos en la gestión educativa de un centro educativo, y como esta incidencia impacta el curso mismo de todo el sistema educativo de un país, siendo el punto clave de cambios trascendentales para el desarrollo de cambios en las políticas públicas educativas, las intervenciones a favor de la calidad educativa y la disminución de la inequidad educativa.

Así las cosas, el liderazgo educativo se proyecta como un valor agregado que se debe gestionar, administrar y antes que nada saber ejecutar de forma transversal en la organización 
educativa, donde su resultado es materializa al visualizar el cumplimiento de metas, el alcance de objetivos y el compromiso de los equipos de trabajo que lidera el director educativo, dejando claro, que el director educativo por sí solo no puede alcanzar las metas propuestas para su centro educativo, sino que requiere la participación activa de sus colaboradores, demostrando una relación directa con la gestión de la cultura organizacional encaminada al cumplimiento conjunto de metas.

Las trasformaciones que se generan en el entorno educativo, obedecen en gran medida al liderazgo de los directores educativos y al nivel de participación que tienen las comunidades educativas frente a los cambios que se experimentan en los diferentes contextos, por lo tanto, la gestión en un centro educativo y de un sistema educativo en general, recaen en gran medida en la labor que los directores educativos tienen para con sus comunidades y participantes, por lo tanto el liderazgo es identificable como el elemento transformador en estos resultados, y a la vez como el epicentro de múltiples cambios que redundan en la adecuada o no gestión educativa (Vázquez, 2017).

Si bien es cierto que la labor del director educativo tiene impacto en toda la comunidad educativa, es claro identificar que la administración educativa debe concentrar su gestión en los siguientes cuatro elementos, los cuales si bien no son los únicos, tienden a representar en gran medida los alcances desde la gestión y liderazgo educativo, como son (Fernandes y Guimãraes, 2013):

a) La gestión del cambio en la organización educativa.

b) Incidencia de la comunicación en el liderazgo educativo.

c) El manejo de conflictos en el entorno académico.

d) La cultura organizacional en el centro educativo.

\section{LA GESTIÓN DEL CAMBIO EN LA ORGANIZACIÓN EDUCATIVA}

El concepto de -cambio- con el paso del tiempo se ha consolidado como un elemento a considerar en la agenda de todo tipo de organizaciones, es por esto que en la organización educativa el cambio se consolida como el punto de partida con el cual los programas, planes y proyectos que se conciben en el escenario educativo, logran tener un alto impacto que se traduce en propuestas educativas contextualizadas y acordes a las necesidades de las poblaciones que hacen parte de un comunidad educativa (Argos y Ezquerra, 2014).

Ahora bien, al analizar la participación del cambio en la administración en un centro educativo, esta responsabilidad recae en el director educativo no solo por su función para con el cumplimiento de metas, sino a la vez, como una posibilidad de encontrar acciones para materializar la planeación estratégica que continuamente se está gestionando desde la alta dirección de una institución de educación; la realidad del director educativo se ve enfrentada a comprender con 
detenimiento las múltiples barreras y dificultades por las cuales los miembros de sus comunidades educativas están atravesando (Hué, 2008), por lo tanto cuando se revisa el escenario latinoamericano, no resulta desconocido el evidenciar que los estereotipos culturales e ideológicos, tengan una marcada tendencia a asociar la dirección educativa a una estructura rígida de control, al conjunto sistemático de acciones concentradas a ejercer intimidación y hasta temor.

En Latinoamérica, la dirección de un centro educativo por bastantes años atrás ha guardado cercana relación y similitud con las estructuras de control que se confeccionan en entornos como lo son: el escenario militar, penitenciario y de guarda civil, razón por la cual directa e indirectamente, en la sociedad latinoamericana concibe la gestión de un centro educativo como un escenario de adiestramiento, acondicionamiento y adopción de posturas, las cuales para muchos pueden llegar a ser las más adecuadas y por lo tanto, las más aceptadas por la sociedad (Pareja, 2009).

A pesar de saber que el cambio, es un término que en el siglo XXI está siendo utilizado por la mayoría de la ciudadanía, representa un reto para quien asume la dirección de un centro educativo, donde quien se presenta como director educativo debe comprender el significado tácito del quehacer de quien ejerce la dirección en una institución de educación, involucrando a todo el equipo de trabajo que hace parte de un centro educativo (Gento y Pina, 2011).

Desde la perspectiva del director educativo para con su equipo directo de trabajo como lo son: directores de área, directores de departamento, coordinadores, líderes de procesos, etc., el cambio se gestiona a partir del involucramiento de cada miembro en la toma de decisiones y así mismo, en asignar responsabilidades conjuntas con las cuales se desarrollen habilidades y destrezas únicas que hacen que el equipo de trabajo construya confianza en sí mismos y se disminuya drásticamente el temor a lo desconocido y hacia explorar nuevas formas y métodos de abordar las situaciones cotidianas (Medina-Alvarado, I, 2019).

De igual forma, el director educativo para todo su equipo de trabajo directo e indirecto, debe construir entornos de participación que favorezcan lo siguiente (Nail y Monereo, 2018):

- El intercambio de saberes y por ende, la construcción colectiva de respuestas a soluciones coyunturales, logrando de este modo que los equipos de colaboradores intercambien opiniones y de forma conjunta propongan alternativas de respuesta.

- El desarrollo de habilidades conjuntas, las cuales se traducen en estrategias organizacionales que aportan en la construcción de una cultura organizacional que sobrevive a las adversidades y se articula desde todas las áreas que la comprende, lo cual hace que la organización educativa adquiera una identidad y sello único.

- Iniciativas por asumir riesgos, si bien es claro que los riesgos conllevan a situaciones caóticas en cualquier tipo de organización, en un centro educativo la gestión de riesgos se traduce en 
explorar nuevas formas de desarrollar los procesos, innovar y fomentar la creatividad, dejando claro que todo riesgo se corre en el marco de lo permitido y desde el alcance que tiene la naturaleza misma de un centro de educación.

\section{INCIDENCIA DE LA COMUNICACIÓN EN EL LIDERAZGO EDUCATIVO}

La comunicación en el escenario del siglo XXI, se ha consolidado como un foco clave de las relaciones humanas, y es que precisamente desde la comunicación y el intercambio de información a través de las múltiples herramientas y canales que se tienen en la actualidad, es que el mundo se está moviendo, ante lo cual las organizaciones educativas no son la excepción y deben identificar en sus procesos de comunicación, los elementos que la conforman para establecer mecanismos de gestión y orientación hacia lo que se espera (Fernández y Garmendia, 2002).

De la mano de la planeación estratégica y la administración educativa, la comunicación en un centro educativo, deben ser una prioridad para quien está al frente de la dirección de un centro de educación, razón por la cual es clave identificar que el administrar los canales de comunicación en un centro educativo se traducen en iniciativas que se evidencian en:

- Diagnosticar los mecanismos de comunicación, esta labor incluye una ardua tarea para el director y líder educativo, quien a partir de su labor y relaciones con sus colaboradores, debe comprender dos escenarios clave del manejo de la información como son: a) canales de comunicación formales, y b) canales de comunicación informales (Antúnez y Pino, 2014).

Cuando se alude en un centro de educación a un canal de comunicación formal, se hace referencia a los mecanismos conocidos por todos, y que tradicionalmente se pueden llegar a traducir como los comúnmente usados, ahora bien, al carácter de formal es asignado a este tipo de comunicación porque logra que todos los miembros de un equipo de trabajo lo asocien como el canal de comunicación con la información más confiable y en cierta medida la información más clara para toda la organización.

De igual forma, al hacer referencia a un canal de comunicación informal al interior de un centro de educación, resulta necesario comprender como la información que no es comunicada a través de un canal de comunicación formal, aun así, si es comunicada y posiblemente esa información que se comparte desde los comentarios en los pasillos, de forma coloquial en un almuerzo o simplemente desde una intención mal intencionada por afectar la integridad de otro, si tiene afectaciones en el clima y la cultura organizacional de una institución de educación, denominada como comunicación informal, expresado esto, el director educativo desde la mirada antiamericana asume una tarea titánica, con al cual sus acciones deben dar cuenta de entornos de interacción en los que sus colaboradores, tiene la confianza, valores y principios, con los cuales 
hacen uso de la información clara, veraz y antes que nada con la intención de comunicarla para favorecer la organización en conjunto y no a intereses individuales (Ulloa y Rodriguez, 2014).

El diagnóstico de los mecanismos de comunicación, con total seguridad le representa al líder, un aprendizaje para sí mismo desde una perspectiva alejada directamente de su labor como director, y con la cual puede intervenir estos canales para hacer un uso adecuado y óptimo de la información que fluye al interior de un centro educativo.

- Intervenir los mecanismos de comunicación, el concepto de intervenir desde esta perspectiva de la comunicación en un centro educativo, no tiene en primera instancia la intención de cambiar abruptamente los medios de comunicación y los canales de comunicación ya establecidos, pero si, tiene como finalidad comprender su funcionamiento, de forma tal que el director educativo pueda hacer uso de estos canales a favor del cumplimiento conjunto de metas y alcanzar la materialización de la misión y visión del centro educativo (Sandoval-Estupiñán, 2008).

El intervenir los mecanismos de comunicación en Latinoamérica no es una tarea fácil, pero si necesaria e indispensable en el camino de pretender involucrar a los colaboradores en las decisiones y la asignación de metas, recordando que un centro educativo maneja multiplicidad de procesos que no solo son de competencia pedagógica, sino que engloban el funcionamiento conjunto de todo el aparato administrativo, liderado antes que nada por el director educativo (Medina-Alvarado, 2019).

- Construir desde la comunicación una ventaja competitiva, las organizaciones educativas tienen la labor de cumplir un proyecto educativo institucional PEI, en el cual se consignan los lineamientos institucionales con los cuales una comunidad educativa encuentra las rutas con las cuales se desarrollan los planes, programas y proyectos, en los que toda la comunidad educativa desde su rol participa y aporta, por lo tanto la integración de esta comunidad para con el cumplimiento de las metas, resulta ser más que necesario e indispensable para sobrevivir.

Desde esta mirada y con relación al contexto latinoamericano, no resulta desconocido que la comunicación cobra un rol determinante en la integración de todos los colaboradores y equipo de trabajo de un centro de educación, y precisamente desde esa mirada es que el término ventaja competitiva se acopla a esta situación, toda vez, que si la organización educativa es liderada por un directivo que conoce a cabalidad los mecanismos de comunicación, puede conseguir que sus equipos de trabajo tengan un alto sentido de compromiso y de responsabilidad, lo cual se traduce en altos resultados en cumplimiento y mejores desempeños en los indicadores establecidos (MedinaAlvarado, 2019).

\section{EL MANEJO DE CONFLICTOS EN EL ENTORNO ACADÉMICO}


En el contexto de la globalización en el cual las organizaciones de todo tipo están desarrollando su labor, día a día los conflictos se consolidan como puntos clave para encontrar múltiples posibilidades para innovar y fomentar la creatividad de los equipos de trabajo, situación que hasta hace una década atrás resultaba ser todo lo contrario, donde los conflictos eran identificados como situaciones negativas y que afectaban a las organizaciones; quizás hoy en día en el escenario académico esta percepción se mantiene, salvo que el reto que asume el director educativo, precisamente se consolida al tener que fomentar los conflictos como elementos esenciales para la interacción y la oxigenación de las estructuras ya consolidadas al interior de los centros de educación, potenciado de este modo el intercambio de saberes, la gestión de habilidades blandas y la exploración de nuevos rumbos al sentido mismo del servicio educativo (Barba y Lobato, 2012).

El manejo de conflictos al interior de un centro educativo requiere que el director educativo desarrolle ciertas habilidades para con el manejo de las relaciones labores con su equipo de trabajo, con la comunidad académica interna y la comunidad externa, siendo necesario comprender lo siguiente:

a) Manejo de las relaciones laborales con el equipo de trabajo, esta perspectiva incluye los mecanismos con los cuales el director fomenta el cumplimiento de directrices y parámetros con los cuales se procura el cumplimiento de metas, y a la vez permite estudiar los diferentes tipos de liderazgo que caracterizar la labor del quien está a cargo del futuro de una institución de educación (Gorrochotegui, 2013).

Es importante mencionar que el manejo de las relaciones labores, requiere contar con destrezas relacionadas con la gestión de equipos de trabajo, tales como: empatía, confianza, sencillez, humildad, entre otras; las cuales no alejan al director de su rol como una autoridad representativa de toda la comunidad educativa, todo lo contrario, le faculta como un individuo que genera acciones para hacer que sus equipos de trabajo sean lo suficientemente capaces para interactuar, desde los principios éticos que determinan el trabajo colectivo y mancomunado.

b) Manejo de las relaciones con la comunidad académica interna, la comunidad académica incluye no solo los docentes y estudiantes, sino que de acuerdo con el centro educativo, puede incluir padres de familia, ante lo cual estas relaciones deben denotar alto sentido de compromiso para con el PEI y los lineamientos establecidos por la dirección educativa, de forma tal que se construya una cultura alrededor de lo que significa la vivencia cotidiana de la filosofía institucional.

El director educativo, si bien es cierto que no tiene una interacción constante y directa con la comunidad académica, en Latinoamérica si es identificado como una autoridad de respeto gracias al cual desde sus múltiples compromisos, está procurando que el horizonte institucional se construya (Álvarez M. , 2011). 
Lo cierto de estas relaciones, es identificar que actores como los docentes son identificados como puntos clave de la interacción entre todos los agentes, tanto es que en muchas instituciones educativas la persecución sobre el funcionamiento de un centro educativo, es construido desde la labor que ejerce el docente, dicho en otras palabras el directivo no puede estar en todo lugar atendiendo las necesidades de su comunidad educativa, situación que no es igual para con el caso de los docentes, quienes de forma repetitiva y constante están interactuando con los estudiantes y hasta con los padres de familia; dejando claro que el liderazgo de un directivo no se traduce únicamente en la labor directa que este hace, sino en el legado que este construye y como los conflictos cuando se presentan, cuentan con conductos definidos para su atención, de forma tal que la organización educativa adopta una cultura enfocada hacia la atención, gestión y control (Gairín y Mercader, 2018).

c) Manejo de las relaciones con la comunidad externa, al aludir al a comunidad externa se hace referencia a todo el público con el cual las organizaciones educativas construyen relaciones directas a indirectas fuera del contexto académico interno de cada institución educativa, por lo tanto la comunidad externa puede generar conflictos o situaciones complejas que requieren un manejo adecuado por parte del directivo académico y su equipo de trabajo.

Los conflictos que comúnmente se presentan con la comunidad externa, aluden a situaciones adversas en las cuales algún miembro de la comunidad educativa se está viendo afectado, por lo el desafío que asume el directo educativo se consolida en el manejo adecuado de la diplomacia, la comunicación efectiva y el dialogo abierto, toda vez que el curso mismo de una institución de educación es vista por el comportamiento mismo de su director (Sandoval, 2006).

\section{LA CULTURA ORGANIZACIONAL EN EL CENTRO EDUCATIVO}

Un centro educativo al igual que cualquier organización, cuenta con un conjunto de aspectos que caracterizan el entorno en el cual las personas que hacen parte de dicho equipo de trabajo interactúan, es por esto que la cultura organizacional si bien es un concepto que ha nacido del entorno empresarial y ejecutivo, tiene total cabida al identificar que el centro educativo requiere por parte del director educativo el contar con habilidades propias para la gestión de entornos laborales (Carda y Larrosa, 2012).

Para comprender la importancia de fortalecer la cultura organizacional en una institución de educación, basta con comprender el lenguaje no verbal y los mecanismos de participación con que interactúan los miembros de dicha institución, siendo clave este punto de partida para comprender los valores, principios y preceptos con los cuales la organización ha logrado crecer de la mano con al proyecto de vida de cada colaborador, de forma tal, que un director educativo debe comprender la estructura no visible con la cual sus colaboradores comparten entre sí, y como las metas y objetivos 
de la organización educativa son cumplidos desde todos y cada uno de los roles que cada quien tiene definido.

El comprender la cultura organizacional en un centro educativo no es una tarea compleja, pero si toma bastante tiempo su caracterización, razón por la cual las cualidades personales y destrezas con las que cuenta el director educativo, son puntos clave para iniciar el proceso de caracterización, donde se destacan los siguientes elementos a considerar (Pareja, 2009):

a) Identidad de la organización, este aspecto alude al conocimiento y empatía que la dirección de la organización educativa ha forjado para con sus colaboradores, de forma tal que el resultado esperado de esta tarea se resume al encontrar que todo el equipo de trabajo conoce plenamente la organización y de igual forma comunica este conocimiento de forma clara y espontánea.

Una forma clara de emprender la identidad de una organización educativa, se puede medir cuando el lenguaje con el cual un colaborador se refiere a su organización, incluye aspectos que resaltan no solo su labor, sino que contribuyen a crear una imagen amplia y generosa de lo que es dicha organización educativa, adicional este lenguaje tiene impregnada la filosofía misma de este centro educativo; así las cosas, la cotidianidad puede denotar que un colaborador que no está conforme o a gusto con su organización, lo da a conocer desde una conversación corta y superficial sobre su labor en esta (Zabalza-Beraza, y Zabalza-Cerdeiriña, 2012).

Para medir la identidad de la organización educativa al interior de los equipos de trabajo, es necesario conocer en detalle los mecanismos de interacción con los que estos equipos de trabajo comparten y antes que nada gestionar entornos de retroalimentación para construir de forma conjunta esa identidad que se espera sea interiorizada por todos y cada uno de los miembros, dejando a un lado la imposición de una filosofía para dar paso a una filosofía que se vive y se construye día a día.

b) Reconocimientos e incentivos, dentro de la gestión de equipos de trabajo los reconocimientos e incentivos para con la labor bien hecha, son claves para que una cultura organizacional sea fuerte y a la vez coherente con la planeación estratégica que se ha propuesto alcanzar en el corto, mediano y largo plazo; aun así, los centros educativos en cabeza de su director educativo deben estar reevaluando y actualizando los mecanismos con los cuales se reconoce dicha labor (Álvarez, Figueiredo, y Egido, 2000).

En la cultura organizacional de todo centro educativo, es necesario y de suma importancia que el reconocimiento ante los buenos resultados y en general a la labor de todos y cada uno de los integrantes de los equipos de trabajo, sea considerado como un elemento de la agenda del día a día, toda vez, que este ejercicio de reconocimiento de la labor de los colaboradores fortalece las 
relaciones internas de los equipos de trabajo y orienta los esfuerzos de la dirección hacia la consecución de metas, de allí que en un centro educativo sea necesario junto con este reconocimiento e incentivos, el supervisar que no se generen rivalidades internas, sino que se pueda construir un ambiente para reducir las debilidades y aumentar las fortalezas.

c) Sistemas de control, cuando de alude a los sistemas de control resulta clave comprender los parámetros definidos por la dirección educativa y sus diferentes áreas, de forma tal que el control es asociado directamente con el cumplimiento correcto de las indicaciones y orientaciones que son dadas a los equipos de trabajo (Mañú y Goyarrola, 2012).

Al interior de la organización educativa y desde el conocimiento de la cultura organizacional, los sistemas de control contribuyen a comprender de manera enfática la claridad con que una indicación es dada y como a la vez esta es recibida, siendo todo esto reflejado en el resultado que se obtiene (Argos y Ezquerra, 2014); adicional, la evaluación de los sistemas de control tienen incidencia directa en el control de procesos y procedimientos, procurando la participación de todos quienes están directamente relacionados en un determinado proceso, evitando de este modo la imposición de controles que pueden estar alejados de la realidad que se pretende controlar, y como situación positiva se pretenden buscar escenarios para que las indicaciones dadas, el control y seguimiento a estas denoten resultados esperados y exitosos.

d) Estructuras de poder, el término poder por mucho tiempo se ha empleado correcta e incorrectamente en muchos de los aspectos de la cotidianidad misma, ahora bien, las estructuras de poder en un centro educativo se asocian a quienes toman las decisiones y como estas decisiones impactan el alcance de objetivos para con toda la comunidad educativa, es así como las estructuras de poder a interior de un centro educativo aportan significativamente en la intención de gestionar la cultura organizacional y los elementos no tangibles que afectan positiva o negativamente la gestión de equipos de trabajo (Gairín y Mercader, 2018).

El director educativo debe ser lo suficientemente capaz para comprender como las estructuras de poder se compartan al interior de su centro educativo, siendo claro que el director educativo no puede estar en todo lugar o en toda situación a resolver, pero si debe dejar clara su postura y forma de actuar para con quienes se les ha entregado autoridad en el cumplimiento de las tareas de cada área de trabajo, dicho en otras palabras el director educativo debe comunicar con vehemencia la forma en que se toman las decisiones y como estas obedecen no a un capricho de momento o personal, sino a la necesidad de garantizar el mejor manejo de las relaciones desde la dirección (Barba y Lobato, 2012).

Así las cosas, se resalta la importancia de comprender a fondo la forma en que se gestionan las relaciones labores en una organización, y como la cultura organizacional es un elemento transversal que incluye todas las áreas de la organización y a todos los colaboradores de un equipo 
de trabajo, afirmando de este modo que el liderazgo y la gestión educativa si o solo si, se genera desde la gestión de personas y la asignación de valor a quienes conforman una comunidad educativa.

\section{CONCLUSIONES}

Es claro mencionar que la labor del directivo académico desde el contexto latinoamericano debe estar a cargo de un individuo con cualidades y destrezas únicas, que a la vez sean muy bien definidas, razón por la cual se concluye en primera instancia que este sujeto es un protagonista sobresaliente al interior de una comunidad educativa, encargado de supervisar y controlar toda la operación de una institución de educación, donde esta labor no la puede hacer por sí solo, sino que debe confeccionar un equipo de trabajo altamente competitivo y calificado, que le favorezca en aunar esfuerzos para alcanzar las metas propuestas y visualizar un horizonte organizacional que sea comprendido por todos y cada uno de sus colaboradores.

De igual forma se concluye, que el liderazgo educativo desde el escenario latino para que alcance un aporte destacado, y antes que nada sea lo suficientemente representativo en una organización educativa, debe procurar la gestión del cambio como un motor de innovación y una puerta infinita de creatividad, la cual requiere indispensablemente la participación conjunta de todos los miembros de la organización, dejando a un lado los preceptos establecidos en torno al temor al cambio y al encontrar nuevas formas de realizar las labores.

Para el director educativo es clara la necesidad de comprender a fondo los mecanismos de comunicación con los cuales interactúan sus colaboradores, y no solo esto, sino a la vez comprender los canales de comunicación con que él interactúa con sus colaboradores, por lo tanto de esta iniciativa depende gran parte del éxito de su labor y la faculta para identificar en las situaciones conflictivas o en las que nacen los conflictos, puntos de partida para innovar y proyectar el cambio a favor de la optimización de los recursos con que se ejecutan las labores.

A la vez, es concluyente que la labor del director educativo va mucho más allá de la operación misma de toda la organización, y es precisamente desde esta perspectiva que el director educativo al contar con un equipo de trabajo altamente comprometido y que demuestra sentido de pertenencia, visibiliza la posibilidad de construir una cultura organizacional enfocada a crecimiento conjunto, desde la cual se procura que el proyecto de vida de cada uno de sus colaboradores este alineado estratégicamente con el plan de crecimiento de la institución de educación, resaltando que las organizaciones educativas construyen sus ventajas competitivas desde las personas que la conforman.

\section{BIBLIOGRAFÍA}


Álvarez, A., Figueiredo, I., y Egido, I. (2000). Organización y gestión de los centros educativos en Iberoamérica. Madrid: Organización de Estados Iberoamericanos (OEI).

Álvarez, M. (2011). Liderazgo compartido: buenas prácticas de dirección escolar. Madrid: Wolters Kluwer España.

Antúnez, S., y Pino, M. (2014). Organización de centros educativos en la sociedad del conocimiento. Madrid: Difusora Larousse - Alianza Editorial.

Argos, J., y Ezquerra, P. (2014). Liderazgo y educación. Santander: PUbliCan Editorial de la Universidad de Cantabria.

Barba, A., y Lobato, O. (2012). Instituciones de educación superior, políticas públicas y organización. México D.F.: Editorial Miguel Ángel Porrúa.

Carda, R., y Larrosa, F. (2012). La organización del centro educativo: manual para maestros. San Vicente: ECU - Editorial Club Universitario.

Fernandes, E., y Guimãraes, D. (2013). Proyectos educativos y sociales: planificación, gestión, seguimiento y evaluación. Madrid: Narcea Ediciones.

Fernández, J., y Garmendia, J. (2002). Cultura de la organización y centro educativo. Madrid: Universidad Complutense de Madrid.

Gairín, J., y Mercader, C. (2018). Liderazgo y gestión del talento en las organizaciones. Madrid: Wolters Kluwer España.

Gento, S., y Pina, J. (2011). Gestión, dirección y supervisión de instituciones y programas de tratamiento educativo de la diversidad. Madrid: UNED - Universidad Nacional de Educación a Distancia.

Gorrochotegui, A. (2013). El docente líder: ideas para la auto-mejora continua. Buenos Aires: Miño y Dávila.

Hué, C. (2008). Bienestar docente y pensamiento emocional. Madrid: Wolters Kluwer España.

Mañú, J., y Goyarrola, I. (2012). Docentes competentes: por una educación de calidad. Madrid: Narcea Ediciones.

Medina-Alvarado, I. (2019). Desarrollo Educativo, Económico y Social, aportes de la Teoría del Capital Humano en América Latina. Revista Caribeña de Ciencias Sociales, 1-7.

Medina-Alvarado, I.L. (2019). El liderazgo y la gestión educativa a partir de la comunicación al interior de las organizaciones educativas. Revista Caribeña de Ciencias Sociales, 1-7.

Nail, O., y Monereo, C. (2018). Gestión y liderazgo en el ámbito de la convivencia escolar. Santiago de Chile: RIL editores.

Pareja, J. (2009). Liderazgo y conflicto en las organizaciones educativas. Revista Educación y Educadores, 137-152.

Sandoval, L. (2006). El ser y el hacer de la organización educativa. Revista Educación y Educadores, 33-54.

Sandoval-Estupiñán, L. (2008). Institución educativa y empresa: dos organizaciones humanas distintas. Bogotá D.C.: Editorial EUNSA y Universidad de La Sabana. 
Ulloa, J., y Rodriguez, S. (2014). Liderazgo escolar y desarrollo profesional de docentes: aportes para la mejora de la escuela. Santiago de Chile: RIL editores.

Vázquez, E. (2017). La inspección y supervisión de los centros educativos. Madrid: UNED Universidad Nacional de Educación a Distancia.

Zabalza-Beraza, M, y Zabalza-Cerdeiriña, M. (2012). Innovación y cambio en las instituciones educativas. Santa Fé: Homo Sapiens Ediciones. 